PROCEEDINGS OF THE

AMERICAN MATHEMATICAL SOCIETY

Volume 126, Number 12, December 1998, Pages 3581-3587

S $0002-9939(98) 04594-8$

\title{
A CHARACTERIZATION OF UNIFORM CONTINUITY FOR VOLTERRA EQUATIONS IN HILBERT SPACES
}

CARLOS LIZAMA

(Communicated by Palle E. T. Jorgensen)

\begin{abstract}
We show that the norm continuity of the resolvent for a Volterra equation of scalar type is equivalent to the decay to zero of a holomorphic operator family along some imaginary axis.
\end{abstract}

\section{INTRODUCTION}

Let $X$ be a complex Banach space. In this work we consider the following Volterra equation of scalar type

$$
u(t)=\int_{0}^{t} a(t-s) A u(s) d s+f(t), \quad t \in J:=[0, T],
$$

where $A$ is a closed linear, densely defined operator in $X$, and $a \in L_{l o c}^{1}\left(\mathbb{R}_{+}\right)$a scalar kernel. A continuous function $u: J \rightarrow X$ is called a strong solution of (1.1) on $J$ if $u(t) \in D(A)$ for all $t \in J, A u(t)$ is continuous, and (1.1) holds on $J$. The basic concept concerning (1.1) is that of well-posedness which is the direct extension of the corresponding notion usually employed for the abstract Cauchy problem (of first order)

$$
\dot{u}(t)=A u(t), \quad u(0)=u_{0} .
$$

In [4] it is shown that well-posedness is equivalent to the existence of a resolvent $\{S(t)\}_{t \geq 0} \subseteq \mathcal{B}(X)$ for (1.1), i.e. a strongly continuous family of bounded linear operators in $X$ which commutes with $A$ and satisfies the resolvent equation

$$
S(t) x=x+\int_{0}^{t} a(t-s) A S(s) x d s, \quad t \geq 0, \quad x \in D(A) .
$$

The resolvent is the central object to be studied in the theory of Volterra equations; it corresponds to the strongly continuous semigroup generated by $A$ in the special case $a(t)=1$, i.e. for (1.2). The importance of the resolvent $S(t)$ is shown

Received by the editors May 28, 1996 and, in revised form, April 21, 1997.

1991 Mathematics Subject Classification. Primary 47D06; Secondary 47A50.

Key words and phrases. Volterra equations, resolvent equation, uniform continuity, Laplace transform.

This research was done while the author was visiting at the Mathematisches Institut, Universität Tübingen supported by the Alexander von Humboldt Foundation.

(C) 1998 American Mathematical Society 
by the variation of parameters formula

$$
u(t)=S(t) f(0)+\int_{0}^{t} S(t-s) \dot{f}(s) d s, \quad t \in J,
$$

where $f \in W^{1,1}(J ; X)$.

Due to the time invariance of (1.1), Laplace transform methods can be employed. Formally the Laplace transform $H(\lambda)=\hat{S}(\lambda)$ of the resolvent is represented by

$$
H(\lambda)=(\lambda-\lambda \hat{a}(\lambda) A)^{-1} .
$$

This formula leads to a characterization of Hille-Yosida type for existence of an at most exponentially growing resolvent for (1.1) in terms of properties of the holomorphic operator family $H(\lambda)$. For a general exposition of the theory, we refer to J. Prüss [4].

In this article we characterize the uniform continuity of the resolvent $S(t)$ for (1.1) on Hilbert spaces in terms of (1.5). We show that the norm continuity of $S(t)$ for $t>0$ is equivalent to the decay to zero of $H(\lambda)$ along some imaginary axis. For the special case $a(t)=1$ and $A$ generator of a $C_{0}$-semigroup $T(t)$ in $H$, we have $S(t)=T(t)$ and the result reduces to a characterization of eventually norm continuous semigroups obtained recently by P. You [5] (see also [1] and [3]).

\section{A characterization in Hilbert spaces}

Let $H$ be a complex Hilbert space, $A$ a closed linear unbounded operator in $H$ with dense domain $D(A)$, and $a \in L_{l o c}^{1}\left(\mathbb{R}_{+}\right)$a scalar kernel $\neq 0$. We consider the Volterra equation

$$
u(t)=f(t)+\int_{0}^{t} a(t-s) A u(s) d s, \quad t \in J
$$

where $f \in C(J ; H), J:=[0, T]$.

Since (2.1) is a convolution equation on the halfline it is natural to employ the Laplace transform for its study. Besides the standing assumptions on $a(t)$ and $A$ we therefore suppose that $a(t)$ is Laplace transformable, i.e. there is $\omega \in \mathbb{R}$ such that $\int_{0}^{\infty} e^{-\omega t}|a(t)| d t<\infty$. But we also have to restrict the class of resolvents.

We recall that $S(t)$ is called exponentially bounded of type $(M, w)$, if there are constants $M \geq 1$ and $\omega \in \mathbb{R}$ such that

$$
\|S(t)\| \leq M e^{\omega t}, \text { for all } t \geq 0 .
$$

We will also need the following definition from [4].

Definition 2.1. Let $a \in L_{l o c}^{1}\left(\mathbb{R}_{+}\right)$be Laplace transformable and $k \in \mathbb{N}$. $a(t)$ is called $k$-regular if there is a constant $C>0$ such that

$$
\left|\lambda^{n} \hat{a}^{(n)}(\lambda)\right| \leq C|\hat{a}(\lambda)|
$$

for all $\operatorname{Re} \lambda>\omega, 0<n \leq k$.

Convolutions of $k$-regular kernels are again $k$-regular. Moreover, integration and differentiation are operations which preserve $k$-regularity as well. See [4], p. 70.

Our main result in this article is the following characterization.

Theorem 2.2. Let $A$ be a closed linear unbounded operator in a Hilbert space $H$ with dense domain $D(A)$ and let $a \in L_{l o c}^{1}\left(\mathbb{R}_{+}\right)$satisfy $\int_{0}^{\infty} e^{-\omega t}|a(t)| d t<\infty$ for some $\omega \in \mathbb{R}$. Assume (2.1) admits a resolvent $S(t)$ of type $(M, w)$, and $a(t)$ is 2-regular. Then the following conditions are equivalent. 
(a) $(S(t))_{t \geq 0}$ is continuous in $\mathcal{B}(H)$ for $t>0$,

(b) $\lim _{|\mu| \rightarrow \infty}\left\|H\left(\mu_{0}+i \mu\right)\right\|=0$ for some $\mu_{0}>\omega$.

The above theorem reduces for the case $a(t) \equiv 1$ to the following result of $\mathrm{P}$. You [5].

Corollary 2.3. Let $A$ be the generator of a strongly continuous semigroup $T(t)$ on a Hilbert space $H$. Then the following conditions are equivalent.

(a) $(T(t))_{t>0}$ is norm continuous for $t>0$,

(b) $\lim _{|\mu| \rightarrow \infty}\left\|R\left(\mu_{1}+i \mu ; A\right)\right\|=0$ for some $\mu_{1}>\omega$.

Uniform continuity of the resolvent $S(t)$ has a number of important consequences. For example, $u(t)$ defined by (1.4) in general is a solution of (1.1) if Af $\in W^{1,1}(J ; H)$ or $f=a * g$ with $g \in W^{1,1}(J ; H)$; if $S(t)$ is $\mathcal{B}(H)$-continuous, then $A f \in B V(J ; H)$ or $f=a * g$ with $g \in B V(J ; H)$ are already sufficient.

Another type of application concerns stability of (1.1) or, in other terms, integrability of $S(t)$ (see [4], section 10).

For example, suppose $b \in L_{l o c}^{1}\left(\mathbb{R}_{+}\right)$satisfies $\int_{0}^{\infty}|b(t)| e^{-\beta t} d t<\infty$ for some $\beta \geq 0$, and assume there is a resolvent $S_{b}(t)$ for

$$
v(t)=g(t)+\int_{0}^{t} b(t-s) A v(s) d s, \quad t \in \mathbb{R}_{+},
$$

such that $\omega_{b}=\varlimsup_{t \rightarrow \infty} t^{-1} \log \left|S_{b}(t)\right|<\infty$.

Let $c(t)$ be a completely positive function with associated creep function

$$
k(t)=\kappa+\omega t+\int_{0}^{t} k_{1}(s) d s, \quad t>0,
$$

where $\kappa, \omega \geq 0, k_{1}(t) \geq 0$ nonincreasing with $\lim _{t \rightarrow \infty} k_{1}(t)=0$, and let $\alpha=\omega+$ $k_{1}\left(0^{+}\right)$. According to [4], Theorem 4.1, there is a resolvent $S_{a}(t)$ for (1.1), where $a \in L_{l o c}^{1}\left(\mathbb{R}_{+}\right)$is defined by $\hat{a}(\lambda)=\hat{b}(1 / \hat{c}(\lambda))$, for Re $\lambda$ sufficiently large.

As a direct consequence of Theorem 2.2 and [4], Theorem 10.4, we obtain the following result.

Theorem 2.4. Let $H$ be a Hilbert space. Suppose the function

$$
g(z, \omega)=w^{-1}(\hat{b}(z) / \hat{b}(z+z \omega)-1)
$$

is analytic at $(\infty, 0)$, let $1 / \kappa+\alpha<\infty$ and assume $\alpha>\omega_{b}$, and $b(t)$ is 2-regular. Then $S_{a}(t)$ is uniformly integrable if $S_{a}(t)$ is integrable and

$$
\lim _{|\mu| \rightarrow \infty}\left\|H_{b}\left(\mu_{0}+i \mu\right)\right\|=0
$$

for some sufficiently large $\mu_{0}$.

In order to prove Theorem 2.2 we will need the following lemmas.

Lemma 2.5. Let $a \in L_{l o c}^{1}\left(\mathbb{R}_{+}\right)$be Laplace transformable and assume that

$$
H(\lambda)=(\lambda-\lambda \hat{a}(\lambda) A)^{-1}
$$

exists for all Re $\lambda>\omega$. Then there are functions $k_{1}(\lambda), k_{2}(\lambda)$ and $h_{1}(\lambda), h_{2}(\lambda)$, $h_{3}(\lambda)$ such that

(i) $H^{\prime}(\lambda)=k_{1}(\lambda) H(\lambda)+k_{2}(\lambda) H(\lambda)^{2}, \operatorname{Re} \lambda>\omega$,

(ii) $H^{\prime \prime}(\lambda)=h_{1}(\lambda) H(\lambda)+h_{2}(\lambda) H(\lambda)^{2}+h_{3}(\lambda) H(\lambda)^{3}, \operatorname{Re} \lambda>\omega$. 
Proof. First, we observe that $\hat{a}(\lambda) \neq 0$ for $\operatorname{Re} \lambda>\omega$ because $A$ is unbounded ([4], p. 43). Next, let $H(\lambda)=p(\lambda)(q(\lambda)-A)^{-1}$ where $p(\lambda):=\frac{1}{\lambda \hat{a}(\lambda)}$ and $q(\lambda):=\frac{1}{\hat{a}(\lambda)}$. Then,

$$
H^{\prime}(\lambda)=\frac{p^{\prime}(\lambda)}{p(\lambda)} H(\lambda)-\frac{q^{\prime}(\lambda)}{p(\lambda)} H(\lambda)^{2}, \quad \operatorname{Re} \lambda>\omega
$$

and

$$
H^{\prime \prime}(\lambda)=\frac{p^{\prime \prime}(\lambda)}{p(\lambda)} H(\lambda)+\left[\frac{2 q^{\prime}(\lambda) p^{\prime}(\lambda)}{p(\lambda)^{2}}-\frac{q^{\prime \prime}(\lambda)}{p(\lambda)}\right] H(\lambda)^{2}-\frac{2 q^{\prime}(\lambda)^{2}}{p(\lambda)^{2}} H(\lambda)^{3}, \quad \operatorname{Re} \lambda>\omega .
$$

Remark. A calculation shows us that

$$
k_{1}(\lambda):=-\left(\frac{1}{\lambda}+\frac{\hat{a}^{\prime}(\lambda)}{\hat{a}(\lambda)}\right), \quad k_{2}(\lambda):=\frac{\lambda \hat{a}^{\prime}(\lambda)}{\hat{a}(\lambda)}, \quad \operatorname{Re} \lambda>\omega,
$$

and

$$
\begin{gathered}
h_{1}(\lambda):=\frac{2 \hat{a}^{\prime}(\lambda)}{\lambda \hat{a}(\lambda)}+\frac{2}{\lambda^{2}}+\frac{2 \hat{a}^{\prime}(\lambda)^{2}}{\hat{a}(\lambda)^{2}}-\frac{\hat{a}^{\prime \prime}(\lambda)}{\hat{a}(\lambda)}, \quad \operatorname{Re} \lambda>\omega, \\
h_{2}(\lambda):=\frac{2 \hat{a}^{\prime}(\lambda)}{\hat{a}(\lambda)}+\frac{\lambda \hat{a}^{\prime \prime}(\lambda)}{\hat{a}(\lambda)}, h_{3}(\lambda):=\frac{-2 \lambda^{2} \hat{a}^{\prime}(\lambda)^{2}}{\hat{a}(\lambda)^{2}}, \operatorname{Re} \lambda>\omega .
\end{gathered}
$$

As a direct consequence of formulae (2.2)-(2.4) we obtain the following:

Lemma 2.6. Let $a \in L_{l o c}^{1}\left(\mathbb{R}_{+}\right)$be Laplace transformable and 2-regular, then there exists a constant $M>0$ such that

(i) $\left|\lambda^{1-j} k_{1+j}(\lambda)\right|<M$ for all $\operatorname{Re} \lambda>\omega ; \quad j=0,1$,

(ii) $\int_{|\mu| \geq N}\left|h_{j}\left(\mu_{0}+i \mu\right)\right|^{j} d \mu<M$ for all $\mu_{0}>\omega$ and $N>1 ; j=1,2$,

(iii) $\operatorname{Sup}_{|\mu| \geq N}\left|h_{3}\left(\mu_{0}+i \mu\right)\right|<M$ for all $\mu_{0}>\omega$ and $N>1$.

We will need also the following result from [1].

Lemma 2.7. Let $X$ be a Banach space and let $R:[0, \infty) \rightarrow X$ be a function which is continuous for $t>0$. Moreover, assume that there exist $M>0, \omega \in \mathbb{R}$ such that $\|R(t)\| \leq M e^{\omega t}$. Then the Laplace transform $\hat{R}(\lambda):=\int_{0}^{\infty} e^{-\lambda t} R(t) d t$ exists for all $\lambda \in \mathbb{C}$ satisfying $\operatorname{Re} \lambda>\omega$ and

$$
\lim _{|\mu| \rightarrow \infty} \hat{R}\left(\mu_{0}+i \mu\right)=0
$$

for every $\mu_{0}>\omega$.

We can now prove our main result. The proof is inspired by the proof of $\mathrm{O}$. El Mennaoui and K.-J. Engel [1] for the semigroup case.

Proof of Theorem 2.2. (a) implies (b). We use Lemma 2.7 choosing $X=\mathcal{B}(H)$ and $R(t)=S(t)$.

(b) implies (a). Let $x \in H$ be fixed. Because $\left\|S(t) e^{-\mu_{0} t} x\right\| \leq M e^{\omega_{0} t}\|x\|$ with $\omega_{0}:=\omega-\mu_{0}<0$, the function $t \rightarrow \chi_{[0, \infty)}(t) e^{-\mu_{0} t} S(t) x$ is in $L^{2}(\mathbb{R} ; H)$, where $\chi_{[0, \infty)}(t)$ denotes the characteristic. 
Since $H$ is a Hilbert space, by Plancherel's theorem the Fourier transform is a unitary operator on $L^{2}(\mathbb{R} ; H)$. Thus we obtain

$$
\mathcal{F}\left(\chi_{[0, \infty)}(\cdot) e^{\left.-\mu_{0} \cdot S(\cdot)\right)}=H\left(\mu_{0}+i \mu\right) x\right.
$$

Hence,

$$
S(t) e^{-\mu_{0} t} x=\frac{1}{2 \pi} \int_{-\infty}^{\infty} e^{i \mu t} H\left(\mu_{0}+i \mu\right) x d \mu
$$

for $t>0$ and each $x \in H$. Clearly, the resolvent $S(t)$ is continuous in $\mathcal{B}(H)$ for $t>0$ if and only if $S_{\mu_{0}}(t):=S(t) e^{-\mu_{0} t}$ is continuous in $\mathcal{B}(H)$ for $t>0$. Next, note that for $x \in D(A)$ we have

$$
H\left(\mu_{0}+i \mu\right) x=\hat{a}\left(\mu_{0}+i \mu\right) H\left(\mu_{0}+i \mu\right) A x+\frac{1}{\mu_{0}+i \mu} x
$$

Observe that $\hat{a}\left(\mu_{0}+i \mu\right) \rightarrow 0$ as $|\mu| \rightarrow \infty$ by the Riemann-Lebesgue lemma. In particular $\lim _{|\mu| \rightarrow \infty} H\left(\mu_{0}+i \mu\right) x=0$. Using this and integrating by parts in (2.5) we obtain

$$
S_{\mu_{0}}(t) x=\frac{-1}{2 \pi t} \int_{-\infty}^{\infty} e^{i \mu t} H^{\prime}\left(\mu_{0}+i \mu\right) x d \mu
$$

for all $x \in D(A), t>0$. Next, by Lemma 2.5(i) and Lemma 2.6(i) we also get $\lim _{|\mu| \rightarrow \infty} H^{\prime}\left(\mu_{0}+i \mu\right) x=0$. Using this and again integration by parts we obtain the formula

$$
S_{\mu_{0}}(t) x=\frac{1}{\pi t^{2}} \int_{-\infty}^{\infty} e^{i \mu t} H^{\prime \prime}\left(\mu_{0}+i \mu\right) x d \mu
$$

for all $x \in D(A), t>0$.

Next we show that the operator family $\left(t^{2} S_{\mu_{0}}(t)\right)$ is continuous in $\mathcal{B}(H)$ for $t>0$. In fact, formula (2.6) shows

$$
\begin{aligned}
\left\|t^{2} S_{\mu_{0}}(t) x-s^{2} S_{\mu_{0}}(s) x\right\|=\frac{1}{\pi}\left\|\int_{-\infty}^{\infty}\left(e^{i \mu t}-e^{i \mu s}\right) H^{\prime \prime}\left(\mu_{0}+i \mu\right) x d \mu\right\| \\
\leq \quad \frac{1}{\pi}\left\|\int_{|\mu| \geq N}\left(e^{i \mu t}-e^{i \mu s}\right) H^{\prime \prime}\left(\mu_{0}+i \mu\right) x d \mu\right\| \\
\quad+\frac{1}{\pi} \int_{|\mu| \leq N}\left|e^{i \mu t}-e^{i \mu s}\right|\left\|H^{\prime \prime}\left(\mu_{0}+i \mu\right) x\right\| d \mu \\
=: I_{1}(N)+I_{2}(N) .
\end{aligned}
$$


Let $\epsilon>0$. We show that $I_{1}(N)<\epsilon\|x\|$ for $N$ sufficiently large. To this end take $x^{*} \in H$ and observe that by Lemma 2.5 (ii) we have

$$
\begin{aligned}
& \frac{1}{2}\left|\left\langle\int_{|\mu| \geq N}\left(e^{i \mu t}-e^{i \mu s}\right) H^{\prime \prime}\left(\mu_{0}+i \mu\right) x d \mu, x^{*}\right\rangle\right| \\
& \leq \int_{|\mu| \geq N}\left|\left\langle h_{1}\left(\mu_{0}+i \mu\right) H\left(\mu_{0}+i \mu\right) x, x^{*}\right\rangle\right| d \mu \\
&+\int_{|\mu| \geq N}\left|\left\langle h_{2}\left(\mu_{0}+i \mu\right) H\left(\mu_{0}+i \mu\right)^{2} x, x^{*}\right\rangle\right| d \mu \\
&+\int_{|\mu| \geq N}\left|\left\langle h_{3}\left(\mu_{0}+i \mu\right) H\left(\mu_{0}+i \mu\right)^{3} x, x^{*}\right\rangle\right| d \mu \\
& \leq S u p_{|\mu| \geq N}|| H\left(\mu_{0}+i \mu\right) \|\left(\left(\int_{|\mu| \geq N}\left|h_{1}\left(\mu_{0}+i \mu\right)\right| d \mu\right)\left\|x|| \mid x^{*}\right\|\right. \\
& \quad+\left(\int_{|\mu| \geq N}|| H\left(\mu_{0}+i \mu\right) x \|^{2} d \mu\right)^{1 / 2}\left(\int_{|\mu| \geq N}\left\|h_{2}\left(\mu_{0}+i \mu\right) x^{*}\right\|^{2} d \mu\right)^{1 / 2} \\
& \quad+\operatorname{Sup}_{|\mu| \geq N}\left|h_{3}\left(\mu_{0}+i \mu\right)\right|\left(\int_{|\mu| \geq N}\left\|H\left(\mu_{0}+i \mu\right) x\right\|^{2} d \mu\right)^{1 / 2} \\
&\left.\times\left(\int_{|\mu| \geq N}\left\|H\left(\mu_{0}+i \mu\right)^{*} x^{*}\right\|^{2} d \mu\right)^{1 / 2}\right)
\end{aligned}
$$

where we used the Cauchy-Schwartz and Hölder inequalities. Next, by the Plancherel theorem for the Hilbert space valued Fourier transform (see [2], Lemma 2) and Lemma 2.6(ii) and (iii), we get the estimate

$$
\begin{aligned}
& \leq \operatorname{Sup}_{|\mu| \geq N}\left\|H\left(\mu_{0}+i \mu\right)\right\|\left(M\|x\|\left\|x^{*}\right\|+\left(2 \pi M \int_{0}^{\infty}\left\|S_{\mu_{0}}(t) x\right\|^{2} d t\right)^{1 / 2}\left\|x^{*}\right\|\right. \\
& \left.+\quad M\left(2 \pi \int_{0}^{\infty}\left\|S_{\mu_{0}}(t) x\right\|^{2} d t\right)^{1 / 2}\left(2 \pi \int_{0}^{\infty}\left\|S_{\mu_{0}}(t)^{*} x^{*}\right\|^{2} d t\right)^{1 / 2}\right) .
\end{aligned}
$$

Since $\left(S_{\mu_{0}}(t)\right)_{t \geq 0}$ and the adjoint $\left(S_{\mu_{0}}(t)^{*}\right)_{t \geq 0}$ are exponentially bounded of type $\left(M, \omega_{0}\right)$ for some $\omega_{0}<0$, there exists a constant $C>0$ such that

$$
2 \pi \int_{0}^{\infty}\left\|S_{\mu_{0}}(t) x\right\|^{2} d t \leq C^{2}\|x\| \quad \text { and } \quad 2 \pi \int_{0}^{\infty}\left\|S_{\mu_{0}}(t)^{*} x^{*}\right\|^{2} d t \leq C^{2}\left\|x^{*}\right\| .
$$

Combining this with the above estimate we obtain a constant $K>0$ such that

$$
\begin{aligned}
I_{1}(N) & =\operatorname{Sup}_{|| x^{*}|| \leq 1}\left|\left\langle\int_{|\mu| \geq N}\left(e^{i \mu t}-e^{i \mu s}\right) H^{\prime \prime}\left(\mu_{0}+i \mu\right) x d \mu, x^{*}\right\rangle\right| \\
& \leq 2 K \operatorname{Sup}_{|\mu| \geq N}|| H\left(\mu_{0}+i \mu\right)\|\| x \| .
\end{aligned}
$$

Since $\lim _{|\mu| \rightarrow \infty}\left\|H\left(\mu_{0}+i \mu\right)\right\|=0$ there exists $N>0$ such that

$$
2 K \operatorname{Sup}_{|\mu| \geq N}|| H\left(\mu_{0}+i \mu\right) \|<\epsilon
$$

which yields the desired estimate $I_{1}(N)<\epsilon\|x\|$ for every $x \in D(A)$. 
In order to estimate $I_{2}(N)$ we observe that $\left|e^{-i \alpha}-1\right|^{2}=4 \sin ^{2}(\alpha / 2), \alpha \in \mathbb{R}$. Therefore, for the above fixed $N$ we have

$$
\begin{aligned}
& \int_{|\mu| \leq N}\left|e^{-i \mu(s-t)}-1\right|\left\|H^{\prime \prime}\left(\mu_{0}+i \mu\right) x\right\| d \mu \\
& \quad \leq 2 \int_{|\mu| \leq N}\left|\sin \left(\frac{(s-t) \mu}{2}\right)\right|\left\|H^{\prime \prime}\left(\mu_{0}+i \mu\right) x\right\| d \mu \\
& \quad \leq|s-t| N \int_{|\mu| \leq N} \| H^{\prime \prime}\left(\mu_{0}+i \mu\right) x|| d \mu .
\end{aligned}
$$

Using these estimates for $I_{1}(N), I_{2}(N)$ and the fact that $D(A)$ is dense in $H$ yields $\left\|t^{2} S_{\mu_{0}}(t)-s^{2} S_{\mu_{0}}(s)\right\|<2 \epsilon$ for all $|s-t|<\delta$. This completes the proof.

\section{ACKNowledgments}

The author thanks the referee for suggestions and improvements on the subject of this paper.

\section{REFERENCES}

[1] O. El-Mennaoui and K.-J. Engel, On the characterization of eventually norm continuous semigroups in Hilbert spaces, Arch. Math. 63 (1994), 437-440. MR 95h:47053

[2] G. Greiner and R. Nagel, On the stability of strongly continuous semigroups of positive operators in $L^{2}(\mu)$, Ann. Scuola Norm. Sup. Pisa Ser. (4), 10 (1983), 257-262. MR 85b:47044

[3] J. Liang and T. Xiao, Norm continuity (for $t>0$ ) of propagators of arbitrary order abstract differential equations in Hilbert spaces, J. Math. Anal. Appl. 204 (1996), 124-137. MR 97h:34077

[4] J. Prüss, Evolutionary Integral Equations and Applications, Birkhäuser-Verlag, Basel, Boston, Berlin, 1993. MR 94h:45010

[5] P. You, Characteristic conditions for a $C_{0}$-semigroup with continuity in the uniform operator topology for $t>0$ in Hilbert space, Proc. Amer. Math. Soc. 116 (1992), 991-997. MR 93b: 47083

Department of Mathematics, University of Santiago de Chile, Casilla 307, Correo 2, Santiago, Chile

E-mail address: clizama@fermat.usach.cl 